
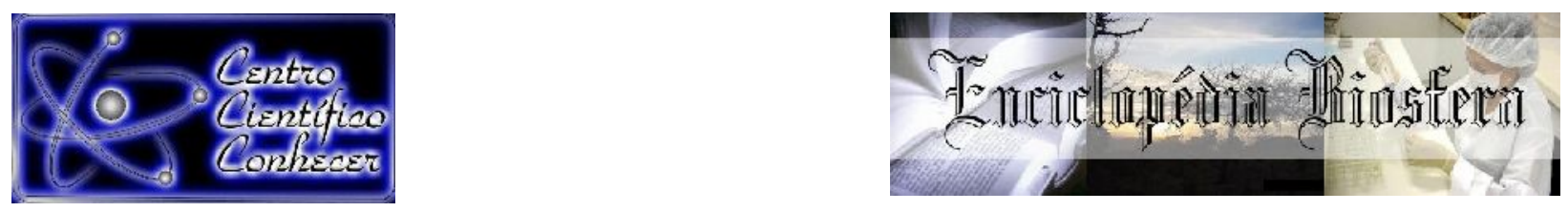

\title{
QUEBRA DE PRODUTIVIDADE E PERÍODO ÓTIMO DE CULTIVO PARA A CULTURA DO MILHO NO SEMIÁRIDO CEARENSE
}

Danilo Batista Nogueira ${ }^{1}$, Alfredo Mendonça de Sousa ${ }^{2}$, José Israel Pinheiro ${ }^{3}$, Lucas de Sousa Oliveira ${ }^{3}$, Alexsandro Oliveira da Silva ${ }^{4}$

${ }^{1}$ Bacharel em Agronomia, Doutorando em Engenharia Agrícola/Universidade Federal do Ceará (UFC), Fortaleza-CE, Brasil.

${ }^{2}$ Bacharel em Agronomia, Doutorando em Engenharia Agrícola/Universidade Federal do Ceará (UFC), Fortaleza-CE, Brasil. E-mail: alfredomendonca121@gmail.com.

${ }^{3}$ Bacharel em Agronomia, Doutorando em Ciências do Solo/Universidade Federal do Ceará (UFC), Fortaleza-CE, Brasil.

${ }^{4}$ Professor Doutor do Curso de Agronomia da Universidade Federal do Ceará (UFC), Fortaleza-CE, Brasil.

Recebido em: 15/05/2020 - Aprovado em: 15/06/2020 - Publicado em: 30/06/2020 DOI: 10.18677/EnciBio_2020B55

\begin{abstract}
RESUMO
A estimativa de produtividade e a definição dos melhores períodos de cultivo constituem-se em fatores preponderantes na obtenção de indicadores técnicos e econômicos satisfatórios para a condução do milho de sequeiro no semiárido. Logo, objetivou-se estimar a produtividade do milho de sequeiro em diferentes cenários pluviométricos e indicar o período adequado para seu cultivo no município de Crateús-Ce, analisando a quebra de produtividade da cultura e suas causas em três cenários pluviométricos. Obteve-se séries históricas de precipitação e temperatura máxima e mínima do ar, que foram agrupadas em períodos de dez dias (decêndios) e os anos de coleta classificados em cenários de ano seco, normal e chuvoso, usando a metodologia de distribuição gama incompleta. A partir destes dados calculou-se o balanço hídrico e foi definido as melhores épocas de semeadura do milho, considerando o período cujo valores da evapotranspiração relativa (ETr/ETp) foram maiores que 0,6 na floração e enchimento dos grãos em mais de $50 \%$ dos anos da série histórica e cuja temperatura média estava entre $15,5{ }^{\circ} \mathrm{C}$ e $33^{\circ} \mathrm{C}$ durante todo o ciclo da cultura. Concluiu-se que em Crateús o manejo ainda é bastante ineficiente e contribui mais que o déficit hídrico na quebra de produtividade em anos normais e chuvosos. A janela de plantio ficou definida a partir de 20/01 a 10/03 para anos chuvosos, de 20/02 até o final de fevereiro para anos normais e não é recomendado seu cultivo em anos secos, cuja queda de produtividade é total em função do déficit hídrico.
\end{abstract}

PALAVRAS-CHAVE: balanço hídrico, cenários pluviométricos, planejamento agrícola, Zea mays L.

\section{BREAKDOWN OF PRODUCTIVITY AND OPTIMUM CULTIVATION PERIOD FOR MAIZE CULTURE IN THE SEMIARID CEARENSE}

\section{ABSTRACT}

The estimation of productivity and the definition of the best growing periods are important factors in obtaining satisfactory technical and economic indicators for the management of rainfed corn in the semiarid region. Therefore, the objective was to estimate the productivity of rainfed corn in different rainfall scenarios and indicate the appropriate period for its cultivation in the municipality of Crateús-Ce, analyzing the ENCICLOPÉDIA BIOSFERA, Centro Científico Conhecer - Goiânia, v.17 n.32; p. 510 2020 
fall in crop productivity and its causes in three rainfall scenarios. Historical series of precipitation and maximum and minimum air temperature were obtained, which were grouped into periods of ten days (ten days) and the collection years classified in dry, normal and rainy year scenarios, using the incomplete range distribution methodology. Based on these data, the water balance was calculated and the best maize sowing times were defined, considering the period in which the relative evapotranspiration values (ETr / ETp) were greater than 0.6 in flowering and grain filling in more than $50 \%$ of the years of the historical series and whose average temperature was between $15.5^{\circ} \mathrm{C}$ and $33^{\circ} \mathrm{C}$ during the entire culture cycle. It was concluded that management in Crateús is still quite inefficient and contributes more than the water deficit to the drop in productivity in normal and rainy years. The planting window was defined from $01 / 20$ to $03 / 10$ for rainy years, from $02 / 20$ until the end of February for normal years and its cultivation in dry years is not recommended, whose fall in productivity is total due to of the water deficit.

KEYWORDS: water balance, rainfall scenarios, agricultural planning, Zea mays L.

\section{INTRODUÇÃO}

Dentre as principais culturas temporárias produzidas no Ceará, destaca-se a produção de grãos como o milho (418.900 t em 2017), feijão (118.800 t em 2017) e arroz em casca (9.700 t em 2017) que juntos são responsáveis por $98,7 \%$ do total de grãos produzidos nesta região (CONAB, 2017). Entre os fatores que influenciam a produção da cultura do milho, o clima e a disponibilidade hídrica destacam-se entre os fatores de maior relevância, sendo a produtividade da cultura afetada significativamente em condições de baixa quantidade de água (CARON et al., 2017; GUO et al., 2017; SHIM et al., 2017; YANG et al., 2017). A necessidade hídrica da cultura do milho varia de acordo com a época, local de cultivo e genótipo utilizado, podendo variar de 250 a $800 \mathrm{~mm}$ ao longo do ciclo de cultivo, e chegar a $900 \mathrm{~mm}$ no ciclo em locais de alta demanda evapotranspirativa (ABDRABBO et al., 2016).

As causas, efeitos e a magnitude de acontecimentos que levam a diminuição na produtividade das culturas vem ganhando forte abordagem por meio de um conceito chamado quebra de produtividade ou yield gap (SENTELHAS et al., 2016). De acordo com Sentelhas et al. (2015), os Yield Gaps mais abordados são três: o total (YGt), por déficit hídrico (YGWD) e devido a deficiências de manejo (YGm); por conseguinte, as quebras serão definidas pelos tipos de produtividade, que no caso, Battisti et al. (2012) as classifica em potencial (PPf), atingível (PA) e real (PR). Nesse aspecto, diversos trabalhos têm sido desenvolvidos avaliando modelos de predição e estimativa de quebra de produtividade e de crescimento em culturas agrícolas em função de diversos fatores, como os relacionados ao clima, genética ou técnicas de manejo (JABEEN et al., 2017; LOPEZ et al., 2017; NEGM et al., 2017; SHRESTHA et al., 2017; SINGH et al., 2017; YIN et al., 2017; PAGANI et al., 2017).

O adequado suprimento hídrico é fator crucial para a obtenção de níveis adequados de produtividade na cultura do milho. Desta forma, dentre as práticas de manejo comuns no cultivo da cultura, a definição do período adequado de semeadura constitui-se em um dos aspectos que mais afetam o potencial produtivo, principalmente sob cultivo de sequeiro. A escolha da data de semeadura é fator preponderante na produtividade obtida.

$\mathrm{Na}$ região semiárida, de modo geral, o milho é cultivado em sistema de sequeiro, sendo plantado no período chuvoso, que no sertão cearense, situa-se entre os meses de janeiro a meados de maio. Devido ao seu porte e área foliar, a cultura consome grande quantidade de água, sendo a disponibilidade desta, fator 
preponderante ao seu desenvolvimento. Na condição de sequeiro, o déficit hídrico se manifesta como um dos principais fatores que levam a quebra de produtividade na cultura do milho. Em pesquisas realizadas no Brasil, na segunda estação de crescimento, foram verificadas quebras de produtividade média de cerca de $2,1 \mathrm{tha}$ 1, obtida em vários locais de cultivo (ANDREA et al., 2018). Em estudos com modelos de simulação envolvendo vários cenários de produção de milho no Brasil, o Atlas global de quebra de produtividade (GYGA, 2015) mostra potencial médio de quebra de produtividade de $4,1 \mathrm{t} \mathrm{ha}^{-1}$, devido ao déficit hídrico, o que é muito elevado, tendo em vista que a média de produtividade da cultura é de aproximadamente $5,0 \mathrm{t} \mathrm{ha}^{-1}$ (CONAB, 2017).

Diante do exposto, objetivou-se com o presente estudo estimar a produtividade da cultura do milho para diferentes cenários pluviométricos (anos secos, normais e chuvosos) e com base nas estimativas realizadas, identificar o período mais adequado para o cultivo da cultura em Crateús-CE, investigando a quebra de produtividade associada a estes três cenários pluviométricos.

\section{MATERIAL E MÉTODOS}

Obteve-se uma série histórica climática com no mínimo 30 anos de coleta de precipitação pluvial e temperatura máxima e mínima do ar, todas em escala diária, a partir do banco de dados da Fundação Cearense de Meteorologia e Recursos Hídricos (Funceme) e do Instituto Nacional de Meteorologia (INMET) referentes ao município de Crateús-CE (Figuras 1), atendendo assim ao período mínimo de 15 anos de dados climáticos diários utilizados na elaboração do zoneamento agrícola de risco climático (BRASIL, 2018). Para a correção dos dados faltantes e inconsistentes adotou-se a metodologia descrita em Vianna e Sentelhas (2014).

FIGURA 1. Localização das estações meteorológicas utilizadas no estudo.

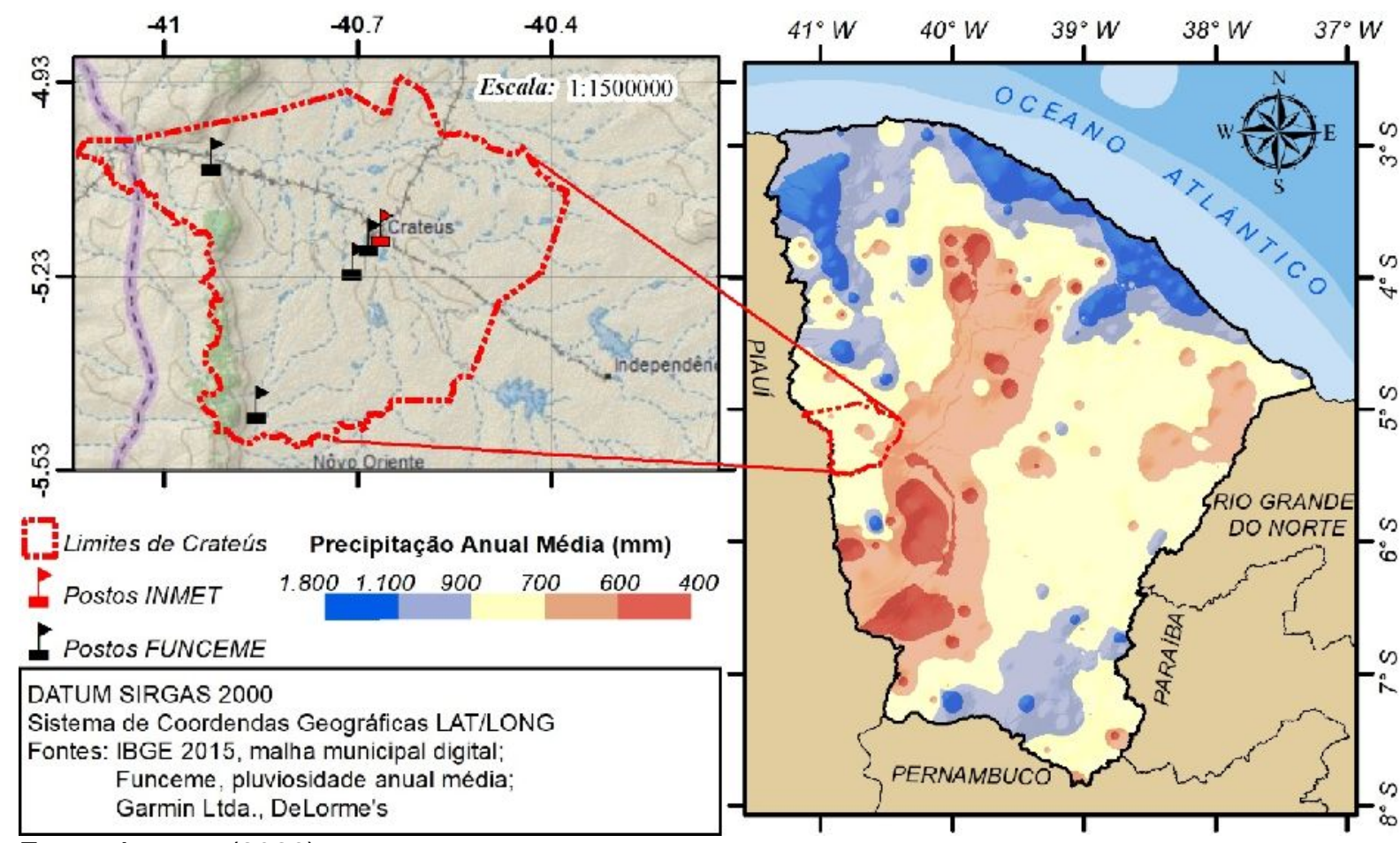

Fonte: Autores (2020) 
Após corrigida, as séries de variáveis climáticas foram agrupadas em períodos de 10 dias (decêndios) e os anos de coletas classificados e agrupados por média em cenários de ano seco, normal e chuvoso para cada uma das estações utilizadas, usando metodologia de distribuição gama incompleta descrita em Thom (1958). Após o agrupamento dos dados foi calculado o balanço hídrico conforme metodologia descrita por Thornthwaite e Mather (1955).

As melhores épocas de semeadura do milho foram consideradas quando em mais de $50 \%$ dos anos a evapotranspiração relativa (ETr/ETp) foi maior que 0,6 na floração e enchimento dos grãos e a temperatura média estava entre 15,5 e $33^{\circ} \mathrm{C}$ durante todo o ciclo da cultura, conforme proposta desenvolvida pelo Ministério da Agricultura, Pecuária e Abastecimento para zoneamento agrícola do milho (BRASIL, 2018). Para simulação utilizou-se características da variedade de milho 'caatingueiro' cujos valores de $\mathrm{kc}$ e fases fenológicas foram obtidos da pesquisa desenvolvida por Souza et al. (2015).

Para estimativa da produtividade real, e a partir da mesma considerar o efeito do déficit hídrico para obter a produtividade atingível (PA), foi utilizado o modelo da Zona Agroecológica - FAO descrito em Doorenbos e Kassam (1979) cujos exemplos de aplicação podem ser verificados em Monteiro e Sentelhas (2017) e Januario et al. (2018). O coeficiente de sensibilidade do milho à deficiência hídrica (ky), utilizado neste modelo, foi obtido da pesquisa de Andrioli e Sentelhas (2009).

Dentre as datas recomendadas à semeadura do milho, utilizou-se aquela com menor valor de evapotranspiração relativa na fase de floração e enchimento de grãos para o cálculo da PPf e da PA, que é estimada com a penalização por déficit hídrico da PPf, obtida conforme o modelo da Zona Agroecológica. Já a PR foi obtida com base em dados processados no SIDRA do Instituto Brasileiro de Geografia e Estatística (IBGE). O yield gap foi determinado pela diferença entre a PR e a PA, representando o yield gap advindo do manejo agrícola (YGM) e pela diferença entre a PPf e a PA, representando o yield gap causado pelo déficit hídrico (YGDH).

\section{RESULTADOS E DISCUSSÃO}

De acordo com as análises feitas, caracterizou-se a quadra chuvosa típica do Nordeste brasileiro conforme as representações gráficas simplificadas do balanço hídrico nos diferentes cenários, a partir da média dos postos utilizados (Figura 2).

FIGURA 2. Extrato do balanço hídrico: cenários de anos chuvosos $(A)$, normais $(B)$ e $\operatorname{secos}(C)$.

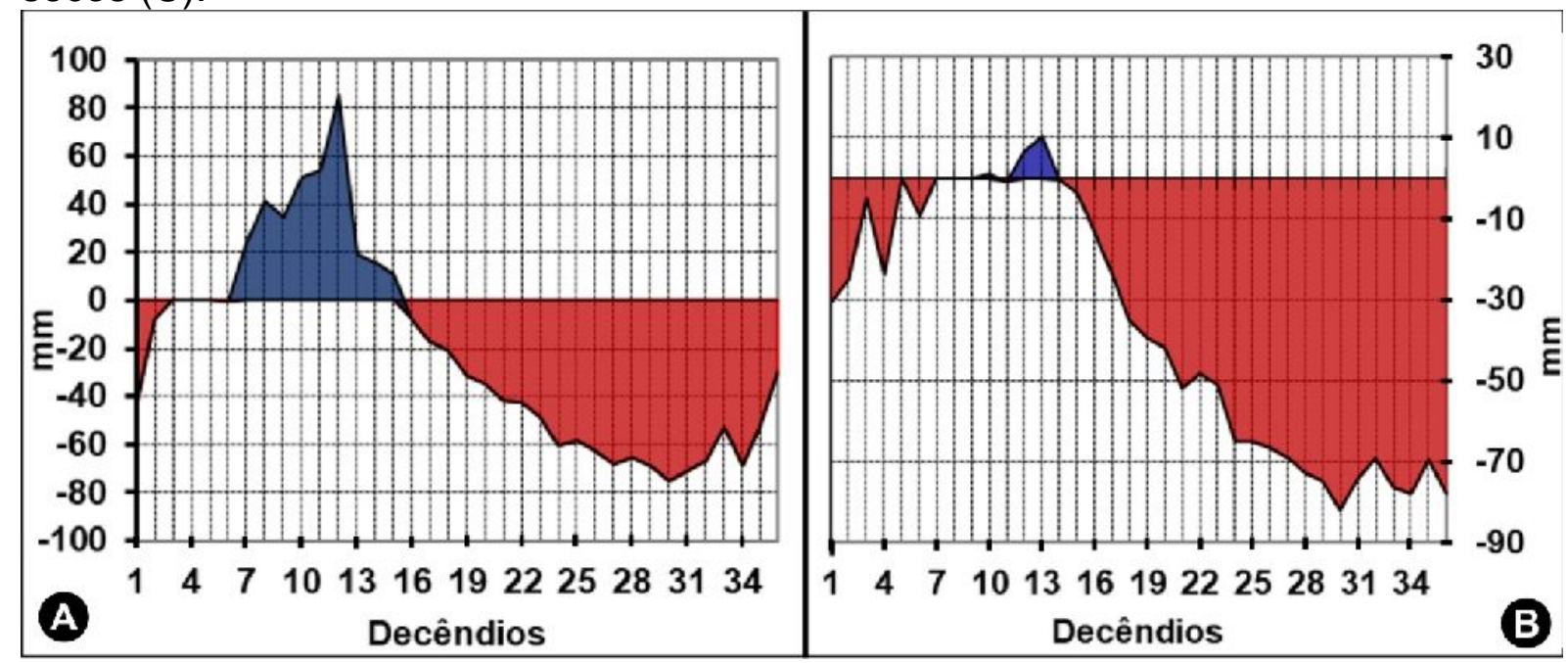




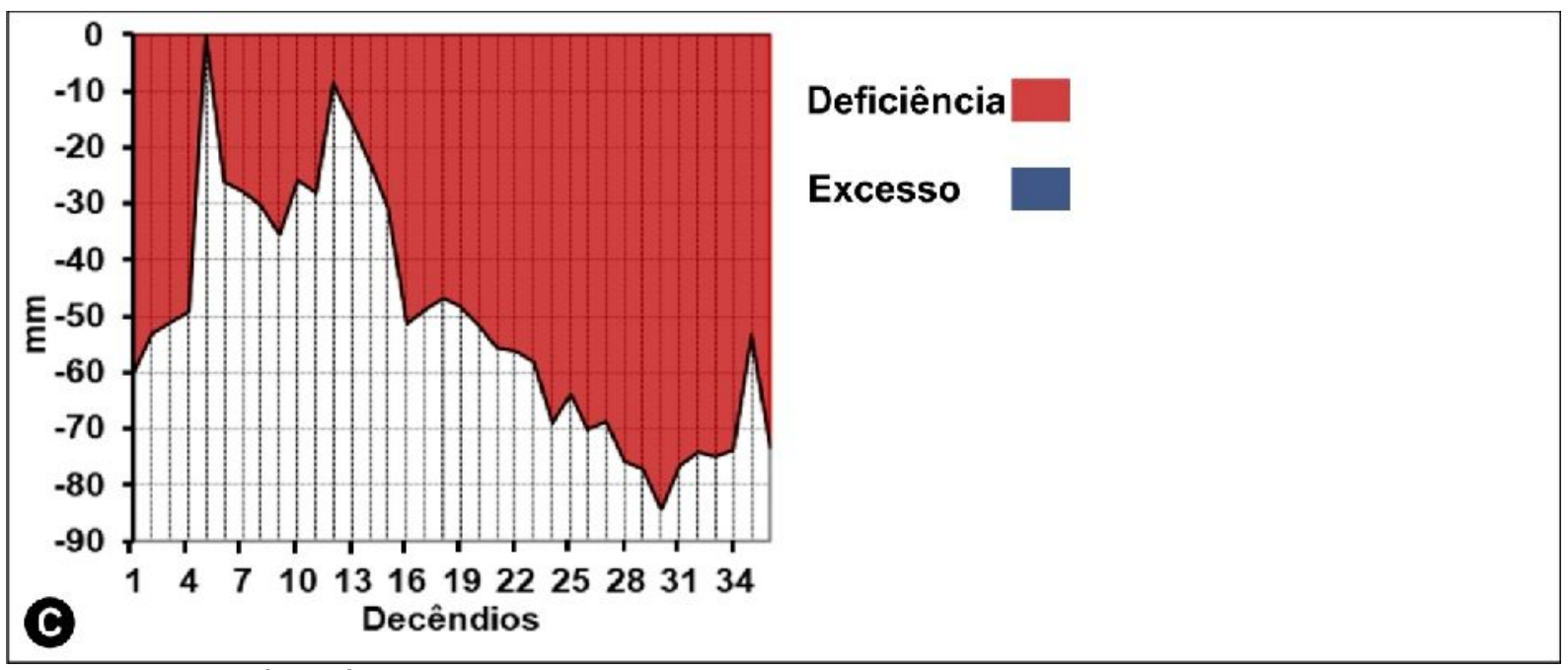

Fonte: Autores (2020)

Fica bem evidente que quanto menor o regime de chuvas, mais concentrado o período chuvoso, e que o mesmo se desloca mais do início do ano para o mês de junho (Figura 2). Isto se reflete diretamente no período para semeio do milho conduzido em sequeiro, que em anos chuvosos começa em 20/01 e em anos normais no dia 20/02 não havendo recomendações no cenário de anos secos. Estes períodos de semeio estão condizentes com as últimas datas recomendadas no zoneamento agrícola de risco climático para a cultura do milho em Crateús-CE, desenvolvida pelo Ministério da Agricultura, Pecuária e Abastecimento (BRASIL, 2018).

As produtividades potenciais obtidas no final da estação recomendada ao semeio do milho foram bem próximas entre os diferentes cenários (Tabela 1). Todavia como era de se esperar a quebra de produtividade foi bem maior nos anos secos. Duarte et al. (2019), estudando quebra de produtividade na cultura do milho no Brasil, verificou, em média, quebra de $52 \%$ da produtividade em função da deficiência hídrica, valor significativamente superior aos verificados no presente estudo. Van Ittersum et al. (2013) afirmam que as grandes diferenças entre estimativas de produtividade potencial e produtividade atingida em estudos globais sob a utilização de modelos genéricos em culturas agrícolas, devem ser validadas localmente visando a obtenção de parâmetros mais consistentes para comparação.

É importante frisar que em condições de agricultura de subsistência, modelo predominante na região semiárida brasileira, a utilização de modelagem de estimativa de produtividade pode subestimar as diferenças de rendimento, como as verificadas na tabela 1 . Melhorias de rendimento devido à mudança de condições de mercado que induzissem a substituição de cultivares tradicionais por cultivares mais resistentes a condições adversas de cultivo por parte dos agricultores, podem constituir-se em um fator de variação nos resultados obtidos em modelagens climáticas desta natureza (AFFHOLDER et al., 2013).

Os resultados demonstram que a PPf foi maior nos anos chuvosos, com valores bem próximos da PA em anos normais (Tabela 1). Entretanto, a janela de plantio é maior para anos chuvosos começando a partir do dia 20/01 até 10/03, enquanto que, em anos normais, a mesma começa no dia 20/02 e vai até o final de fevereiro. Já para anos secos o cultivo do milho em regime de sequeiro não é recomendado para a região, como foi constatado na pesquisa, a quebra de produtividade em virtude do déficit hídrico corresponde a causa total da improdutividade nestes anos, corroborando os resultados obtidos por Duarte et al. 
(2019). No entanto, conforme pode ser visto na Tabela 1, a PR foi um pouco maior que a PA em anos secos, isto deve-se ao cultivo de algumas plantações irrigadas que não são consideradas na metodologia abordada para estimar a PA, mas são dados coletados pelo sistema do IBGE para a PR.

TABELA 1. Produtividades potencial (PPf), atingível (PA) e real (PR) para a cultura do milho em diferentes cenários de precipitação pluviométrica e suas respectivas quebras de produtividade causadas pelo déficit hídrico (YGdh) e pelo manejo agrícola (YGm).

\begin{tabular}{|c|c|c|c|c|c|c|c|c|c|}
\hline \multirow{2}{*}{ Cenário } & \multirow{2}{*}{ Posto } & \multirow{2}{*}{$\begin{array}{l}\text { PPf } \\
\left(\mathrm{t} \mathrm{ha}^{-1}\right)\end{array}$} & \multirow[t]{2}{*}{$\mathrm{PA}$} & \multirow[t]{2}{*}{ PR } & \multicolumn{2}{|l|}{ YGdh } & \multicolumn{2}{|l|}{ YGm } & \multirow{2}{*}{$\frac{\mathrm{YGt}^{1}}{\left(\mathrm{t} \mathrm{ha}^{-1}\right)}$} \\
\hline & & & & & $\left(\mathrm{t} \mathrm{ha}^{-1}\right)$ & $(\%)$ & $\left(\mathrm{t} \mathrm{ha}^{-1}\right)$ & $(\%)$ & \\
\hline Chuvoso & & 5882,1 & 5059,5 & 978,8 & 822,6 & 16,8 & 4080,7 & 83,2 & 4903,3 \\
\hline Normal & $\mathrm{P} 1$ & 5809,8 & 4063,5 & 865,7 & 1746,3 & 35,3 & 3197,8 & 64,7 & 4944,1 \\
\hline Seco & & 5562,1 & 188,9 & 258,0 & 5373,2 & 100,0 & $-69,1$ & 0,0 & 5373,2 \\
\hline Chuvoso & & 5874,3 & 5329,7 & 978,8 & 544,6 & 11,1 & 4350,8 & 88,9 & 4895,4 \\
\hline Normal & P2 & 5808,8 & 5410,1 & 865,7 & 398,8 & 8,1 & 4544,4 & 91,9 & 4943,2 \\
\hline Seco & & 5561,2 & 125,8 & 258,0 & 5435,4 & 100,0 & $-132,2$ & 0,0 & 5435,4 \\
\hline Chuvoso & & 5881,4 & 5120,9 & 978,8 & 760,5 & 15,5 & 4142,1 & 84,5 & 4902,6 \\
\hline Normal & P3 & 5808,5 & 4692,3 & 865,7 & 1116,2 & 22,6 & 3826,7 & 77,4 & 4942,9 \\
\hline Seco & & 5561,0 & 75,7 & 258,0 & 5485,2 & 100,0 & $-182,2$ & 0,0 & 5485,2 \\
\hline Chuvoso & & 5880,5 & 5508,6 & 978,8 & 371,9 & 7,6 & 4529,7 & 92,4 & 4901,6 \\
\hline Normal & $\mathrm{P} 4$ & 5806,9 & 5129,9 & 865,7 & 677,0 & 13,7 & 4264,2 & 86,3 & 4941,2 \\
\hline Seco & & 5559,4 & 129,5 & 258,0 & 5429,9 & 100,0 & $-128,5$ & 0,0 & 5429,9 \\
\hline Chuvoso & & 5881,6 & 5055,5 & 978,8 & 826,1 & 16,8 & 4076,7 & 83,2 & 4902,8 \\
\hline Normal & P5 & 5794,7 & 4372,3 & 865,7 & 1422,5 & 28,9 & 3506,6 & 71,1 & 4929,1 \\
\hline Seco & & 5561,3 & 271,1 & 258,0 & 5290,3 & 99,8 & 13,1 & 99,8 & 5303,3 \\
\hline
\end{tabular}

YGt: Yield gap total.

A quebra de produtividade por ineficiência do manejo agrícola é outro fator que penaliza diretamente a produtividade do milho no Ceará, principalmente pelo fato desta cultura ser conduzida em um sistema de agricultura familiar rústica e pouco tecnificada, geralmente consorciada com feijão (CONAB, 2017). Nos anos normais e chuvosos a expressividade da quebra de produtividade por manejo ineficiente é bem maior que por déficit hídrico (Figura 3).

FIGURA 3. Quebra de produtividade do milho em condições de sequeiro em função do déficit hídrico (YGdh) e do manejo agrícola (YGm).

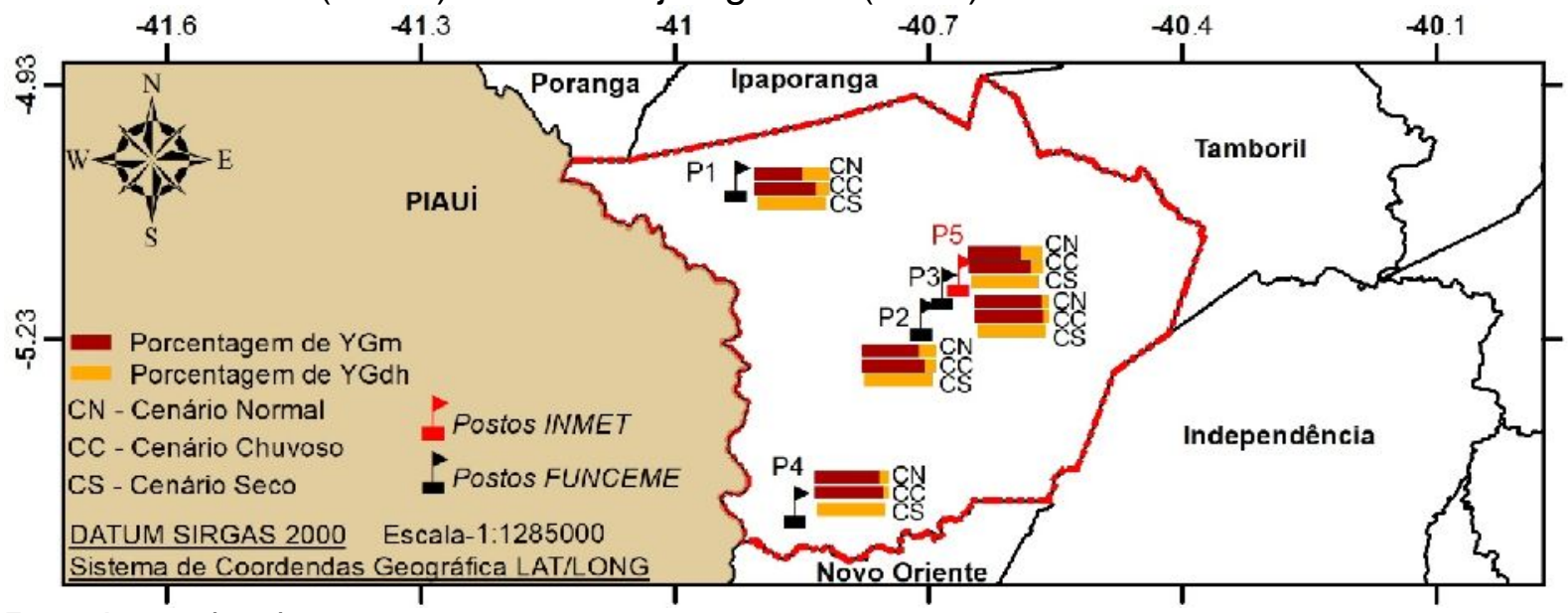

Fonte: Autores (2020) 
Resultados obtidos por Duarte et al. (2019) mostraram que o déficit hídrico causa $53 \%$ das quebras em relação à produtividade potencial, já as perdas de produtividade causadas por déficits de manejo correspondem a mais de $52 \%$ das quebras em relação à produtividade atingível. O déficit hídrico foi responsável por $46 \%$ da quebra de produtividade de milho em trabalho de Hampf et al. (2018), enquanto que, o restante (54\%), foi atribuído ao manejo empregado no sistema de cultivo. Isso mostra o quanto esses fatores são determinantes no que concerne as causas de quebra de produtividade das culturas.

\section{CONCLUSÃO}

A janela de plantio em Crateús-CE ocorre a partir de 20/01 a 10/03 para anos chuvosos e de 20/02 até o final de fevereiro para anos normais. Em anos secos o cultivo do milho em regime de sequeiro não é recomendado para a região.

Tendo em vista que a produção do milho ainda é muito rudimentar no Ceará, sendo conduzida principalmente por pequenos agricultores, trabalhos como este auxilia no melhor planejamento da produção, diminuindo os riscos de quebra de safra. Além disso contribui com uma nova metodologia que busca primeiramente homogeneizar o comportamento classificando os cenários por volume de chuva da estação chuvosa para depois buscar a janela de semeio dentro dos anos.

Em complemento ao que muitas pesquisas demonstram, a baixa produtividade do milho em sequeiro conduzido em regiões semiáridas do Nordeste brasileiro devese, em parte, ao déficit hídrico recorrente que ocorre nestas regiões, em função da má distribuição de chuvas e regime de precipitação com volume abaixo do normal. Todavia, conforme os resultados desta pesquisa, o manejo agrícola ineficiente é também responsável por parte da penalização da produtividade potencial do milho, principalmente, em anos classificados como normal ou chuvoso.

\section{REFERÊNCIAS}

ABDRABBO, M. A. A.; SALEH, S. M.; FARAG, A. A. Water requirements for maize under climate change. Journal of Applied Sciences Research, v. 12, p. 19-28, 2016. Disponível em:

<https://www.researchgate.net/publication/305656464_Water_requirements_for_mai ze_under_climate_change>.

AFFHOLDER, F.; POEYDEBAT, C.; CORBEELS, M.; SCOPEL, E.; TITTONELL, P. The yield gap of major food crops in family agriculture in the tropics: Assessment and analysis through field surveys and modelling. Field Crops Research, v. 143, p. 106118 , 2013.

Disponível em:<https://www.sciencedirect.com/science/article/pii/S0378429012003693>.doi: doi.org/10.1016/j.fcr.2012.10.021

ANDREA, M. C. da S.; BOOTE, K. J.; SENTELHAS, P. C.; ROANELLI, T, L. Variability and limitations of maize production in Brazil: Potential yield, water-limited yield and yield gaps. Agricultural Systems, v. 165, p. 264-273, 2018. Disponível em: <https://www.sciencedirect.com/science/article/abs/pii/S0308521X17311472>. doi: doi.org/10.1016/j.agsy.2018.07.004

ANDRIOLI K, G.; SENTELHAS. P. C. Brazilian maize genotypes sensitivity to water deficit estimated through a simple crop yield model. Pesquisa Agropecuária Brasileira, v. 44, n. 7, p. 653-660, 2009. Disponível em: 
<http://www.scielo.br/scielo.php?script=sci_arttext\&pid=S0100204X2009000700001> doi: doi.org/10.1590/S0100-204X2009000700001

BATTISTI, R.; SENTELHAS, P. C.; PILAU, F. G. Eficiência agrícola da produção de soja, milho e trigo no estado do Rio Grande do Sul entre 1980 e 2008. Ciência Rural, v. $42, \quad$ p. $24-30, \quad 2012.2$ Disponível em:<http://www.scielo.br/scielo.php?script=sci_arttext\&pid=S01034782012000100005>. doi: doi.org/10.1590/S0103-84782012000100005

BRASIL. Ministério da Agricultura, Pecuária e Abastecimento. Anexo I. Parâmetros de Risco Climático in Zoneamento agrícola. 2018. Disponível em: $<$ http://www.agricultura.gov.br/politica-agricola/zoneamentoagricola/portariassegmentadas-por-uf>. Acesso em: 27 dez. 2019.

CARON, B. O.; OLIVEIRA, D. M.; ELLI, E. F.; ELOY, E.; SCHWERZ, F.; SOUZA, V.Q. Elementos meteorológicos sobre características morfológicas e produtivas do milho em diferentes épocas de semeadura. Científica, v. 45, p. 105-114, 2017. Disponível em:<http://cientifica.org.br/index.php/cientifica/article/view/910/565>. doi: doi.org/10.15361/1984-5529.2017v45n2p105-114

CONAB - Companhia Nacional de Abastecimento. 2017. Acompanhamento da safra brasileira de grãos, In: Safra 2016/2017, v.4, Ceará. Disponível em: < http://www.conab.gov.br/OlalaCMS/uploads/arquivos/17_09_12_10_14_36_boletim_ graos_setembro_2017.pdf >. Acesso em: 06 de outubro de $201 \overline{9}$.

DOORENBOS, J.; KASSAM, A. Yield response to water. Irrigation and drainage paper, v. 33, p. 257, 1979.

DUARTE, Y. C. N.; Sentelhas, P. C. Intercomparison and Performance of Maize Crop Models and Their Ensemble for Yield Simulations in Brazil. International Journal of Plant Production, v. 2019, p. 1-13, 2019. Disponível em:<https://link.springer.com/article/10.1007\%2Fs42106-019-00073-5>. doi: doi.org/10.1007/s42106-019-00073-5

GUO, E.; ZHANG, J.; WANG, Y.; ALU, S.; WANG, R.; LI, D.; HA, S. Assessing nonlinear variation of temperature and precipitation for different growth periods of maize and their impacts on phenology in the Midwest of Jim province, China. Theoretical and Applied Climatology, v. 126, p. 1-15, 2017. Disponível em:<https://www.researchgate.net/publication/316078992_Assessing_nonlinear_vari ation_of_temperature_and_precipitation_for_different_growth_periods_of_maize_an d_their_impacts_on_phenology_in_the_Midwest_of_Jilin_Province_China>. doi: $10.1007 / \mathrm{s} 00704-017-2097-\overline{6}$

GYGA. Global Yield Gap Atlas, 2015. Disponível em: <http://www.yieldgap.org/>. Acessado em 18 dez/ 2019

HAMPF, A. C.; CARAUTA, M.; LATYNSKIY, E.; LIBERA, A. A. D.; MONTEIRO, L.; SENTELHAS, P. C.; TROOST, C.; BERGER, T.; NENDEL, C. The biophysical and socio-economic dimension of yield gaps in the southern Amazon - A bio-economic modelling approach. Agricultural Systems, v. 165, p. 1-13, 2018. Disponível em: < 
https://www.sciencedirect.com/science/article/abs/pii/S0308521X17304808>.

doi: doi.org/10.1016/j.agsy.2018.05.009

JABEEN, F.; ASIF, M.; IFTIKHAR, A.; SALMAN, M. Temperature trends and its impact on Zea mays (maize) crop in Faisalabad city through DSSAT-CERES-Maize model. Scientia Agricultarae, v. 17, p. 8-14, 2017. Disponível em: < http://pscipub.com/Journals/Data/JList/Scientia\%20Agriculturae/2017/Volume\%2017/ Issue\%201/2.pdf>. doi: 10.15192/PSCP.SA.2017.1.814

JANUARIO, I. R.; MENEZES, R. H. N. De; BONFIM, O. E. T. Análise de risco climático para semeadura do arroz no Sul do Estado do Maranhão. Revista Brasileira de Geografia Física, v. 11, n. 4, p. 1241-1250, 2018. Disponível em: < https://periodicos.ufpe.br/revistas/rbgfe/article/view/234305/30536>.

LOPEZ, J. R.; ERICKSON, J. E.; ASSENG, S.; BOBEDA, E. L. Modification of the CERES grain sorghum model to simulate optimum sweet sorghumrooting depth for rainfed production oc coarse textured soils in a sub-tropical environment. Agricultural Water Management, v. 181, p. 47-55, 2017. Disponível em: < https://www.sciencedirect.com/science/article/abs/pii/S0378377416304747>. doi: doi.org/10.1016/j.agwat.2016.11.023

MONTEIRO, L. A.; SENTELHAS, P. C. Sugarcane yield gap: can it be determined at nacional level with a simple agrometeorological model?. Crop \& Pasture Science, v. 68 , p. 272-284, 2017. Disponível em:< http://agris.fao.org/agrissearch/search.do?recordID=US201700196213>.

NEGM, L. M.; YOUSSEF, M. A.; JAYNES, D. B. Evaluation of DRAINMOD-DSSAT simulated effects of controlled drainage on crop yield, water balance, and water quality for a corn-soybean cropping system in central lowa. Agricultural Water Management, v. 187, p. 57-68, 2017. Disponível em:<https://www.sciencedirect.com/science/article/abs/pii/S0378377417300938>. doi: doi.org/10.1016/j.agwat.2017.03.010

PAGANI, V.; STELLA, T.; GUARNERI, T.; FINOTTO, G.; BERG, M. V. D.; MARIN, F. R.; ACUTIS, M.; CONFALONIERI, R. Forecasting sugarcane yields using agroclimatic indicators and CaneGro model: A case study in the main production region in Brazil. Agricultural Systems, v. 154, p. 45-52, 2017. Disponível em: <https://www.sciencedirect.com/science/article/abs/pii/S0308521X16308095>. doi: doi.org/10.1016/j.agsy.2017.03.002

SENTELHAS, P. C.; BATTISTI, R.; CÂMARA, G. M. S.; FARIAS, J. R. B.; HAMPF, A. C.; NENDEL, C. The soybean yield gap in Brazil - magnitude, causes and possible solutions for sustainable production. The Journal of Agricultural Science, v. 153, p. 1394-1411, 2015. Disponível em: $<$ https://www.cambridge.org/core/journals/journal-of-agriculturalscience/article/soybean-yield-gap-in-brazil-magnitude-causes-and-possible-solutionsfor-sustainable-production/DB059CC032A27B7C5A07FF896F56E8D5>. doi: doi.org/10.1017/S0021859615000313

SENTELHAS, P. C.; BATTISTI, R.; MONTEIRO, L. A. YIELD GAP - Conceitos, definições e exemplos. Informações Agronômicas. p. 1-4, 2016. Disponível em: < 
http://www.ipni.net/publication/iabrasil.nsf/0/9C05063FB033C24A83258042004C859 4/\$FILE/Page9-12-155.pdf>.

SHRESTHA, S.; CHAMPAGAIN, R.; BABEL, M. S. Quantifying the impact of climate change on crop yield and water footprint on rice in the Nam Oon irrigation Project, Thailand. Science of the Total Environmet, p. 689-699, 2017. Disponível em: < https://www.sciencedirect.com/science/article/pii/S0048969717311270>. doi: doi.org/10.1016/j.scitotenv.2017.05.028

SHIM, D.; LEE, K. J.; LEE, B. W. Response of phenology - and yield-related traits of maize to elevated temperature in a temperate region. The Crop Journal, v. 5, p. 305316 , 2017. Disponível em: <https://www.sciencedirect.com/science/article/pii/S221451411730017X>. doi: doi.org/10.1016/j.cj.2017.01.004

SINGH, P. K.; SINGH, K. K.; BHAN, S. C.; BAXLA, A. K.; SINGH, S.; RATHORE, L. S.; GUPTA, A. Impact of projected climat change on rice (Oryza sativa L.) yield using CERES-rice model in a diferente agroclimatic zones of India. Current Science, v. 112, p. 108-115, 2017.2 Disponível em: $<$ https://www.researchgate.net/publication/315847735_Impact_of_Projected_Climate Change_on_Rice_Oryza_sativa_L_Yield_Using_CERESRice_Model_in_Different_ Agroclimatic_Zones_of_India>. doi: $10.18520 / \mathrm{cs} / \mathrm{v} 112 / \mathrm{i} 01 / 108-115$

SOUZA, L. S. B.; MOURA, M. S. B.; SEDIYAMA, G. C.; SILVA, T. G. F. Requerimento hídrico e coeficiente de cultura do milho e feijão-caupi em sistemas exclusivo e consorciado. Revista Caatinga, v. 28, n. 4, p. 151-60, 2015. Disponível em: <https://periodicos.ufersa.edu.br/index.php/caatinga/article/view/3631/pdf_316>.

THOM, H.C.S. A note on the gama distribuiton. Monthly Weather Review, v. 8, p. 117-122, 1958. Disponível em: < https://journals.ametsoc.org/doi/abs/10.1175/15200493\%281958\%29086\%3C0117\%3AANOTGD\%3E2.0.CO\%3B2>.

THORNTHWAITE, C. W.; MATHER, J. R. The water balance. Publications in Climatology. Drexel Institute of Technology, New Jersey, p. 104, 1955.

VAN ITTERSUM, M. K.; CASSMAN, K. G.; GRASSINI, P.; WOLF, J.; TITTONELL, P.; HOCHMAN, Z. Yield gap analysis with local to global relevance-A review. Field Crops Research, v. 143, p. 4-17, 2013. Disponível em: <https://www.sciencedirect.com/science/article/pii/S037842901200295X>. doi: doi.org/10.1016/j.fcr.2012.09.009

VIANNA, M. S.; SENTELHAS, P. C. Simulação do risco de deficit hídrico em regiões de expansão do cultivo de cana-de-açúcar no Brasil. Revista Pesquisa Agropecuária Brasileira, v. 49, n. 4, p. 237-246, 2014. Disponível em: <http://www.scielo.br/scielo.php?script=sci_arttext\&pid=S0100204X2014000400237> doi: doi.org/10.1590/S0100-204X2014000400001

YANG, H.; GRASSINI, P.; CASSMAN, K. G.; AIKEN, R. M. Improvments to the Hybrid-Maize model for simulating maize yields in harsh rainfed environments. Field 
Crops Research, v. 204, p. 180-190, 2017. Disponível em: <https://www.sciencedirect.com/science/article/pii/S0378429017301727>.

doi: doi.org/10.1016/j.fcr.2017.01.019

YIN, X.; KERSEBAUMB, K. C.; KOLLAS, C.; MANEVSKIA, K.; BABY, S.; BEAUDOIN, N.; et al.,; Performance of process-based models for simulation of grain $\mathrm{N}$ in crop rotation across Europe. Agricultural Systems, v. 154, p. 63-77, 2017. Disponível em:

<https://www.sciencedirect.com/science/article/abs/pii/S0308521X16306394>. doi: doi.org/10.1016/j.agsy.2017.03.005 